\title{
Simple Aesthetic Correction for Patients with Acute Auriculocephalic Angle
}

\author{
Byung Mi Lee, \\ Seok Joo Kang, \\ Hook Sun \\ Department of Plastic and Reconstructive \\ Surgery, Busan Paik Hospital, Inje University \\ College of Medicine, Busan, Korea
}

No potential conflict of interest relevant to this article was reported.

\begin{abstract}
Background: Acute auriculocephalic angle refers to an ear with helix that is spaced closely to the cranium. An increasing number of patients with acute auriculocephalic angle wish to undergo corrective operation for aesthetic purposes. However, there is a paucity of data regarding acute auriculocephalic angle. This paper proposes a treatment protocol for patients with acute auriculocephalic angle.

Methods: We performed a retrospective analysis of patients undergoing acute auriculocephalic angle (4 patients, 6 ears). Patient records were reviewed for demographic data as well as auricular measurements at preoperative, immediate postoperative and final follow-up evaluations.

Results: All of the patients were men with a mean age of 36.5 years (range, 23-52 years). The mean follow-up period was 47.5 months (range, 28-60 months). Postoperative auriculocephalic angle was close to the normal auriculocephalic angle $\left(25^{\circ}-30^{\circ}\right)$ without notable scars. Moreover, the patients had minimal contractions of the skin flaps without any hematoma or relapse.

Conclusion: We propose the following three treatment protocols for patients with acute auriculocephalic angle: the posterior auricular muscle should be sufficiently released, the mastoid area should be augmented using implants, the skin should be repositioned with a superior auricular flap.
\end{abstract}

Keywords: Ear / Surgical procedures / Surgical flaps

\section{INTRODUCTION}

Acute auriculocephalic angle refers to an ear with helix that is spaced closely to the cranium. It is the reverse of prominent ear, the most common type of ear deformity. Acute auriculocephalic angle is distinct from cryptotia in that the skin over the ear cartilage is not continuous with the scalp. Patients commonly complain of small ears based on subjective judgment and inconspicuous profile of the ear from frontal view.

Compared with other otoplastic entities, patients with acute auriculocephalic angle have received little attention. This trend is

\section{Correspondence: Hook Sun}

Department of Plastic and Reconstructive Surgery, Busan Paik Hospital, Inje University College of Medicine, 75 Bokji-ro, Busanjin-gu, Busan 614-735, Korea E-mail: sun443@naver.com

Received February 13, 2015 / Revised March 25, 2015 / Accepted April 2, 2015 changing, however, with more patients expressing desire to undergo operative intervention for aesthetic purposes. However, there is a paucity of report regarding the treatment of acute auriculocephalic angle. In this study, we review a case series of patients undergoing auriculocephalic angle operation and propose a treatment protocol.

\section{METHODS}

\section{Study setting and patient evaluation}

Upon Institutional Review Board approval, a retrospective review was performed for all patients $(\mathrm{n}=4)$ who had undergone acute auriculocephalic angle between January, 2009 and January, 2013. The ear position was evaluated using the perpendicular distance 
extending from the midpoint of helical rim to the mastoid skin (Fig. 1). To avoid the variations in the thicknesses of helices, we used the point along the helical edge that is closest to the scalp as the reference.

Demographic data and auricular measurements were collected from each patient. Auricular measurements were obtained using surgical calipers preoperatively, immediate postoperatively, and final follow-up (Tables 1, 2).

\section{Surgical technique}

The operations were performed under local anesthesia. From the anterior point where the ear diverges from the scalp, a line was drawn along the retroauricular sulcus to the upper midpoint of posterior ear. Next, a line was drawn from the superior crus of the antihelix to the retroauricular sulcus line drawn previously. This resulted in a inverse Y-shaped design (Figs. 2, 3).

After the elevation of the Y-shaped flap, the posterior auricular muscle was identified and carefully incised. This was followed by undermining the upper half of the hair-bearing area over the mastoid, just deep to the periosteum according to the outline of silicone implant to be implanted. A crescent-shaped silicone im-

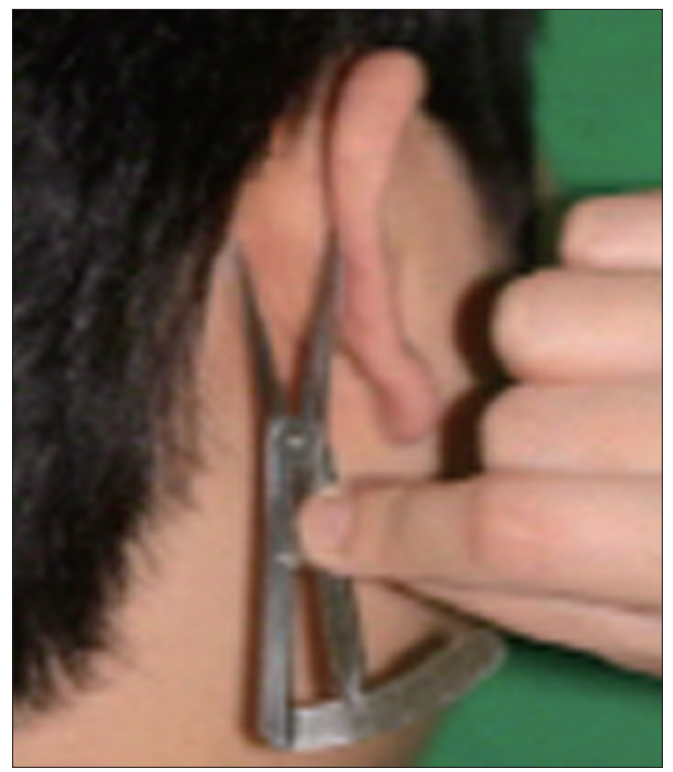

Fig. 1. Location for the measurement of ears. Measurement of the perpendicular distance extending from the helix close to the scalp to the mastoid skin. plant was sculptured to an overall length less than half the ear height. The alloplastic implant was inserted under the periosteum and then fixed with 3-0 nylon suture to prevent implants shifting (Fig. 3). The Y-flap was advanced and closed as a V-flap with 5-0 nylon. A mild compression dressing was maintained for two days without any drain. The wound was protected in additional dressing for additional 7 days.

\section{RESULTS}

\section{Baseline characteristics}

All of the four patients were male, with a mean age of 36.5 years (range, 23-52 years). Two patients underwent bilateral operation, with the remaining two requiring unilateral operations. The patients were followed up during a mean period of 47.5 months (range, 28-60 months).

\section{Postoperative outcomes}

Postoperatively, patients achieved a well-preserved contour of the ear, with auriculocephalic angles close to normal auriculocephalic

Table 1. The shortest perpendicular distance extending from the midpoint of the helical rim to the mastoid skin

\begin{tabular}{lccccc}
$\begin{array}{l}\text { Ear } \\
\text { No. }\end{array}$ & $\begin{array}{c}\text { Age } \\
(\mathrm{yr})\end{array}$ & $\begin{array}{c}\text { Preoperation } \\
(\mathrm{mm})\end{array}$ & $\begin{array}{c}\text { Postoperation } \\
(\mathrm{mm})\end{array}$ & $\begin{array}{c}\text { Final follow- } \\
\text { up }(\mathrm{mm})\end{array}$ & $\begin{array}{c}\text { Follow-up } \\
\text { duration }(\mathrm{mo})\end{array}$ \\
\hline Ear 1. & 38 & 10 & 15 & 15 & 60 \\
\hline Ear 2. & 38 & 11 & 16 & 16 & 60 \\
\hline Ear 3. & 23 & 8 & 17 & 16 & 46 \\
\hline Ear 4. & 23 & 9 & 17 & 17 & 48 \\
\hline Ear 5. & 45 & 8 & 16 & 15 & 43 \\
\hline Ear 6. & 52 & 10 & 21 & 21 & 28 \\
\hline
\end{tabular}

Table 2. Measurement of auriculocephalic angle postoperatively

\begin{tabular}{lccc} 
Ear No. & Age $(\mathrm{yr})$ & Final follow-up $\left(^{\circ}\right)$ & Follow-up duration $(\mathrm{mo})$ \\
\hline Ear 1. & 38 & 25 & 60 \\
Ear 2. & 38 & 26 & 60 \\
\hline Ear 3. & 23 & 26 & 46 \\
Ear 4. & 23 & 26 & 48 \\
Ear 5. & 45 & 25 & 43 \\
Ear 6. & 52 & 28 & 28 \\
\hline
\end{tabular}


angle $\left(25^{\circ}-30^{\circ}\right)$ but without notable scars [1]. There were minimal contraction of skin flaps without hematoma or relapse. Patients were satisfied with postoperative outcomes from both aesthetic and functional perspectives (Figs. 4, 5).

\section{DISCUSSION}

Acute auriculocephalic angled-ear is similar to, but distinct from, cryptotia. It is characterized by the collapse of upper $1 / 3$ of auricle under the temporal skin. As the reverse of prominent ear, it is morphologically characterized by excessive backward curvature and prominence of the antihelix, resulting in a flat-lying shape of the upper ear. Patients with acute auriculocephalic angle commonly complain of small, inconspicuous ears, and wish to undergo otoplastic surgery for aesthetic purposes.
Our clinical series consists of four men, whose ears are more exposed than women, which may explain why men may be more eager to undergo surgery.

Acute auriculocephalic angle is corrected by increasing the angle made between the ear and the cranium to form a natural 3-dimensional structure of the auricle without blunting the inner ear folds.

We propose the following three treatment protocols for patients with acute auriculocephalic angle:

(1) The posterior auricular muscle should be released sufficiently. Guerra et al. [2] reported that $98 \%$ of patients had posterior auricular muscle. There is a linear relationship between the projection of ears and the insertion site in the posterior auricular muscle [3]. Other factors are also involved in the etiology of protruding and lop ears, although intrinsic and extrinsic muscles play major roles in the ultimate shape and position of the auricle. Based
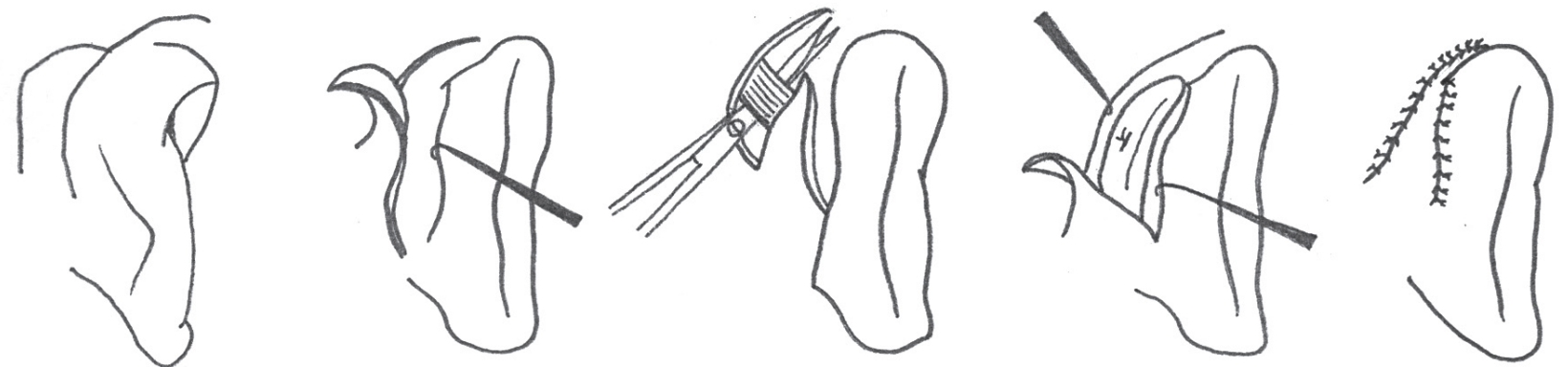

Fig. 2. Schematic diagram of the surgical procedure. Preoperative design of the skin incision was drawn. After elevation of the Y-shaped flap, posterior auricular muscle was incised for free tension. Kidney-shaped implant was inserted under the periosteum. The flap was advanced so that the reverse $\mathrm{Y}$ shape became a reverse $\mathrm{V}$ shape.
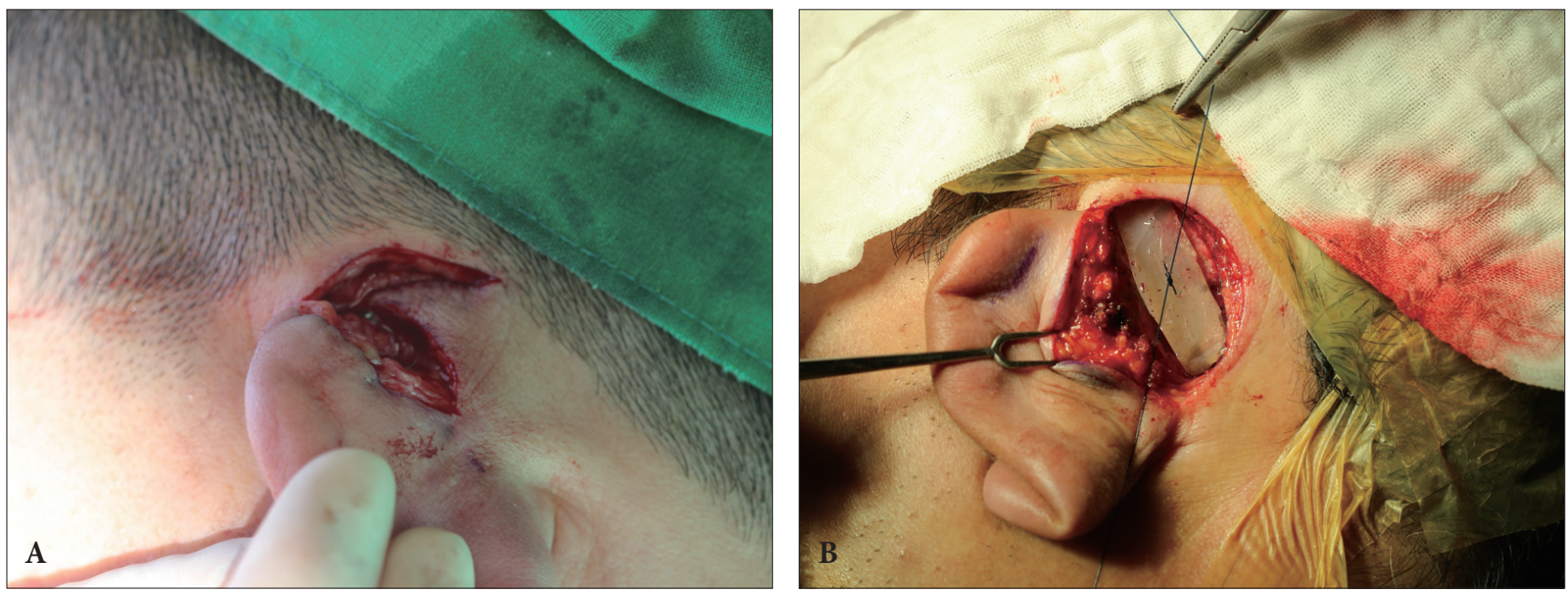

Fig. 3. Intraoperative view of the flap surgery. (A) Y-shaped flap was elevated for advancement. (B) An alloplastic implant was inserted under the periosteum and fixed with Nylon 3-0. 
on these reports, we excised the posterior auricular muscle to release the tension pulling ear towards the scalp and to obtain sufficient space for implant insertion.

(2) The mastoid area should be augmented using implants. Patients with acute auriculocephalic angle have smaller ears, and the upper ear is not readily visible. This is, however, distinct from cryptotia, because the ear cartilage is not buried in the scalp. There is a narrow gap between the scalp and ear cartilage. Therefore, it is necessary to widen the gap by augmentation of the mastoid where the ear diverges from scalp [4]. Currently, use of autologous costal cartilage is the standard treatment method. The major disadvan- tage of using costal cartilage, however, is that it requires a donor site. Alternative approaches include silicone or polyethylene implants, which are easily obtained, manufactured, and easily increase volume. These implants are approved by the Food and Drug Administration, are nontoxic, cause minimal foreign body reactions, and possess appropriate mechanical properties for use in non-load-bearing tissues in the craniofacial region [5]. The implants are placed under the periosteum to prevent protrusion and to allow a natural contour to form.

(3) The skin should be repositioned with a superior auricular flap. For ear surgery, covering skin deficits is an important pro-
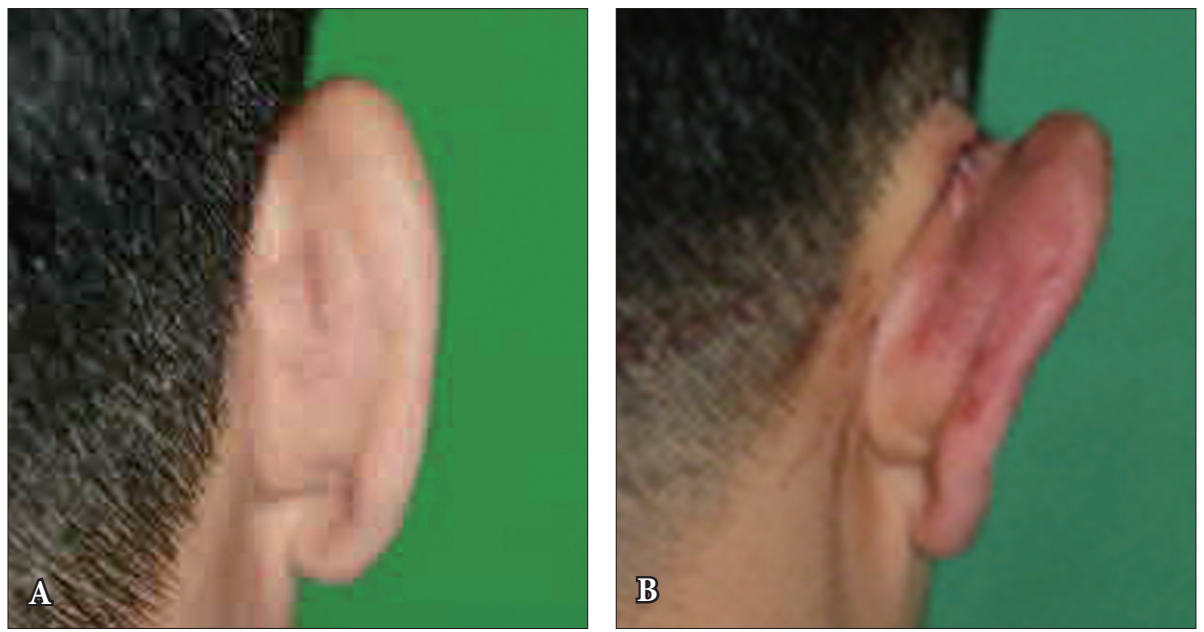

Fig. 4. Case 1. (A) A 23-year-old man had a flat-lying shape of the upper ear. (B) After undergoing Y-V flap surgery, the patient achieved a protrusion of the upper ear.
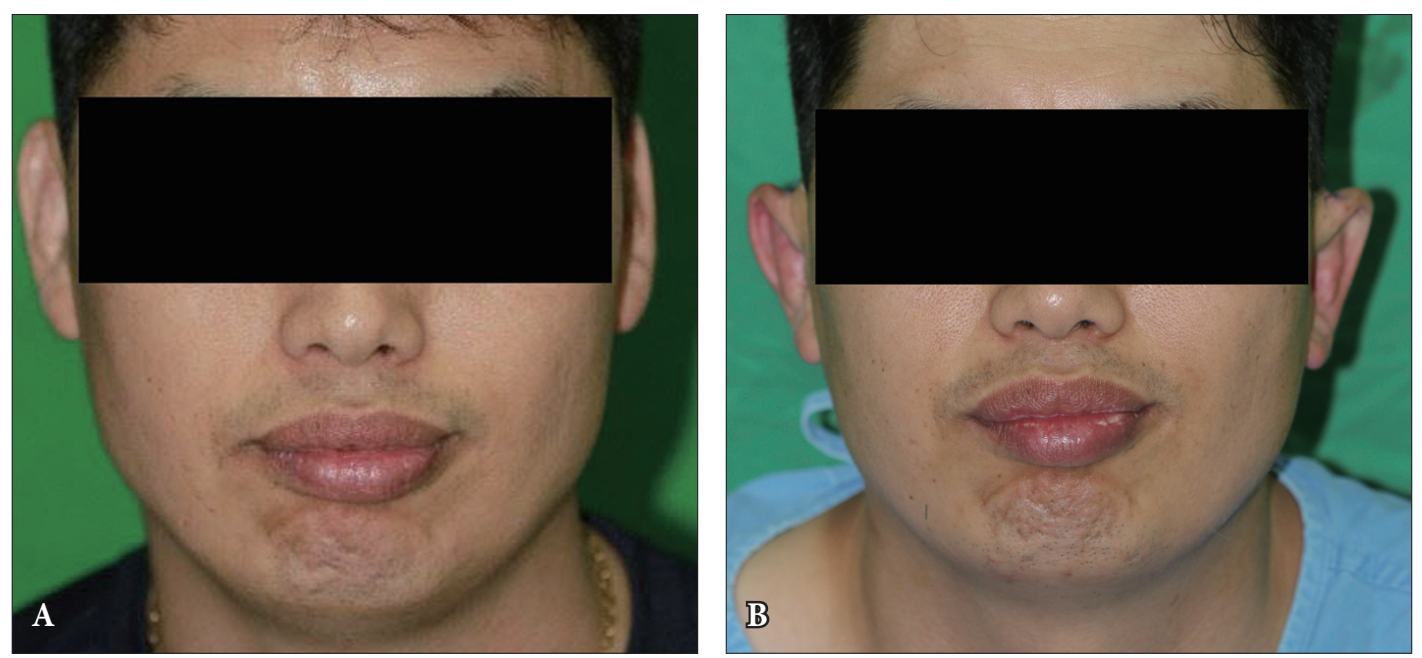

Fig. 5. Case 2. (A) A 38-year-old man had ears that are flat on frontal views. (B) After undergoing superior auricular Y-V flap surgery, increased auriculocephalic angle could make patient's ears visible. 
cess. There are many methods, including local flaps, local flaps with skin grafting, or tissue expansion [6]. The skin graft is disadvantageous because it requires a separate donor site and because there is no appropriate match in color and texture. Because the tissue expansion is a time-consuming process, local flaps are more commonly used for the surgical correction of ear deformities. This is also based on the technical ease of local flap surgery as well as fewer scars [6]. In local flap reconstructions, surgeons commonly perform the V-Y procedure, Z-plasty, rotation flap, and transposition flap. In our series, we used a Y-V flap in the postauricular area to minimize visible scars. By creating a sufficient skin envelope, it is possible to prevent the postoperative skin contraction and to increase the space between scalp and helix.

\section{REFERENCES}

1. Kang JS. Face: reconstruction of the auricle. In: Kang JS, editor. Plastic surgery. 3rd ed. Seoul: Koonja; 2004. p.1464-508.

2. Guerra AB, Metzinger SE, Metzinger RC, Xie C, Xie Y, Rigby PL, Naugle T Jr. Variability of the postauricular muscle complex: analysis of 40 hemicadaver dissections. Arch Facial Plast Surg 2004;6:342-7.

3. Guyuron B, DeLuca L. Ear projection and the posterior auricular muscle insertion. Plast Reconstr Surg 1997;100:457-60.

4. Hwang CM, Lee BK, Green D, Jeong SY, Khang G, Jackson JD, Atala A, Lee SJ, Yoo JJ. Auricular reconstruction using tissue-engineered alloplastic implants for improved clinical outcomes. Plast Reconstr Surg 2014;133:360e-369e.

5. Lee SJ, Broda C, Atala A, Yoo JJ. Engineered cartilage covered ear implants for auricular cartilage reconstruction. Biomacromolecules 2011;12:306-13.

6. Sakamoto Y, Nakajima H, Kishi K, Imanishi N. A new surgical correction of cryptotia with superior auricular myocutaneous flap. J Plast Reconstr Aesthet Surg 2010;63:1995-2000. 